\title{
Robustly chain transitive diffeomorphisms
}

\section{Manseob Lee*}

\section{"Correspondence:}

Imsds@mokwon.ac.kr

Department of Mathematics, Mokwon University, Daejeon, 302-729, Korea

\section{Springer}

\begin{abstract}
In this paper, we discuss the robustly chain transitive set, and show that the robustly chain transitive set is hyperbolic if and only if every periodic points in the set is hyperbolic and has the same index.
\end{abstract}

MSC: 34D23; 34D10; 37C75; 37D20

Keywords: transitive; chain transitive; locally maximal; hyperbolic; dominated splitting

\section{Introduction}

In the theory of dynamical systems one has been to describe and characterize systems exhibiting dynamical properties that are preserved under small perturbations. It is related to the stability theory. In fact, structurally stable systems and $\Omega$-stable systems have been the main objects of interests in the global qualitative theory of dynamical systems and they are characterized as the hyperbolic ones (see [1-4]). Thus, in differentiable dynamical systems, the robustness property is a very interesting topic. Let us consider more details. Let $M$ be a closed $C^{\infty}$ Riemannian manifold, and let $\operatorname{Diff}(M)$ be the space of diffeomorphisms of $M$ endowed with the $C^{1}$-topology. Denote by $d$ the distance on $M$ induced from a Riemannian metric $\|\cdot\|$ on the tangent bundle TM. Let $f \in \operatorname{Diff}(M)$ and $\Lambda$ be a closed $f$-invariant set.

The set $\Lambda$ is transitive if there is a point $x \in \Lambda$ such that $\omega(x)=\Lambda$. Here $\omega(x)$ is the forward limit set of $x$. We say that $\Lambda$ is locally maximal if there is a neighborhood $U$ of $\Lambda$ such that

$$
\Lambda=\bigcap_{n \in \mathbb{Z}} f^{n}(U)
$$

We say that the set $\Lambda$ is robustly transitive if there are a $C^{1}$-neighborhood $\mathcal{U}(f)$ of $f$ and a neighborhood $U$ of $\Lambda$ such that for any $g \in \mathcal{U}(f), \Lambda_{g}(U)=\bigcap_{n \in \mathbb{Z}} g^{n}(U)$ is transitive. Here $\left.f\right|_{\Lambda}$ is transitive means that $f$ is transitive in $\Lambda$. If $\Lambda=M$ then we say that $f$ is robustly transitive. We say that $\Lambda$ is hyperbolic if the tangent bundle $T_{\Lambda} M$ has a $D f$-invariant splitting $E^{s} \oplus E^{u}$ and there exist constants $C>0$ and $0<\lambda<1$ such that

$$
\left\|\left.D_{x} f^{n}\right|_{E_{x}^{s}} ^{s}\right\| \leq C \lambda^{n} \quad \text { and } \quad\left\|\left.D_{x} f^{-n}\right|_{E_{x}^{u}}\right\| \leq C \lambda^{n}
$$

for all $x \in \Lambda$ and $n \geq 0$. If $\Lambda=M$ then we say that $f$ is Anosov. Although $f$ is robustly transitive, we can find that $f$ is not Anosov. In fact, Mañé [5] showed that there exists a diffeo-

(c) 2015 Lee. This article is distributed under the terms of the Creative Commons Attribution 4.0 International License (http://creativecommons.org/licenses/by/4.0/), which permits unrestricted use, distribution, and reproduction in any medium, provided you give appropriate credit to the original author(s) and the source, provide a link to the Creative Commons license, and indicate if changes were made. 
morphism $f$ on the three-dimensional torus $\mathbb{T}^{3}$ that satisfies: there is a $C^{1}$-neighborhood $\mathcal{U}(f)$ of $f$ such that every $g \in \mathcal{U}(f)$ is transitive, but not Anosov.

In [6], Mañé proved that if a diffeomorphism on two-dimensional $C^{\infty}$ manifolds is robustly transitive, then it is hyperbolic, and Díaz et al. [7] proved that if a diffeomorphism on three-dimensional $C^{\infty}$ manifolds is robustly transitive then it is partially hyperbolic. Also, in [8], the authors proved that for $C^{\infty}$ manifolds of any dimension, if a diffeomorphism is robustly transitive, then it admits a dominated splitting.

From the facts, we study the relation between the robustly chain transitive and hyperbolicity. For given $x, y \in M$, we write $x \rightsquigarrow y$ if for any $\delta>0$, there is a finite $\delta$-pseudo orbit $\left\{x_{i}\right\}_{i=0}^{n}(n \geq 1)$ of $f$ such that $x_{0}=x$ and $x_{n}=y$. For any $x, y \in \Lambda$, we write $x \rightsquigarrow \Lambda y$ if $x \rightsquigarrow y$ and $\left\{x_{i}\right\}_{i=0}^{n} \subset \Lambda(n \geq 1)$. We say that the set $\Lambda$ is chain transitive (or, $\left.f\right|_{\Lambda}$ is chain transitive) if for any $x, y \in \Lambda, x \rightsquigarrow \Lambda$. Note that by the definition, a transitive set is a chain transitive set, but the converse is not true (see Example 1.5 in [9]). In this paper, we study robustly chain transitive sets for a diffeomorphism. It is weaker notion of the robustly transitivity. Let $p \in P(f)$ be a hyperbolic point. Denote by $\operatorname{index}(p)=\operatorname{dim} W^{s}(p)$. We say that the set $\Lambda$ is robustly chain transitive if there are a $C^{1}$-neighborhood $\mathcal{U}(f)$ and a neighborhood $U$ of $\Lambda$ such that for any $g \in \mathcal{U}(f), \Lambda_{g}(U)=\bigcap_{n \in \mathbb{Z}} g^{n}(U)$ is chain transitive. Then we have the following.

Theorem 1.1 Let $\left.f\right|_{\Lambda}$ be robustly chain transitive in $U$. Then following conditions are equivalent:

(a) there is a $C^{1}$-neighborhood $\mathcal{U}(f)$ of $f$ such that for any $g \in \mathcal{U}(f)$, any periodic point of $\Lambda_{g}(U)$ is hyperbolic and has the same index;

(b) there is a $C^{1}$-neighborhood $\mathcal{U}(f)$ off such that for any $g \in \mathcal{U}(f), \Lambda_{g}(U)$ is hyperbolic.

\section{Proof of Theorem 1.1}

It is clear that (a) follows from (b) by the local stability of hyperbolic basic set (see Theorem 7.4 in [10]). To prove Theorem 1.1, we show from (a) to (b). We say that $p \in P(f)$ with period $\pi(p)$ is a sink if all the eigenvalues of $D_{p} f^{\pi(p)}$ are less than 1 , and $p \in P(f)$ with period $\pi(p)$ is a source if all eigenvalues of $D_{p} f^{\pi(p)}$ is greater than 1 . The following is the version for diffeomorphisms of the result by Lemma 6 in [11].

Lemma 2.1 Iff $\left.\right|_{\Lambda}$ is chain transitive, then $\left.f\right|_{\Lambda}$ has neither sinks nor sources.

Proof Let $p$ be a sink. Then there exist $\epsilon>0$ and $\lambda<1$ such that if $d(x, p)<\epsilon$ then $d\left(f^{i}(x), p\right)<\lambda d(x, p)$ for all $i \geq 1$. Take $y \in \Lambda$ such that $d(y, p) \geq 2 \epsilon$. For any $\delta>0$, let $\xi=\{p=$ $\left.x_{0}, x_{1}, \ldots, x_{m}=y\right\}(m \geq 1)$ be a $\delta$-pseudo orbit of $f$ such that $x_{i} \in \Lambda$. For simplicity, we may assume that $f(p)=p$. Then we have $d\left(p, x_{1}\right)<\delta$, and $d\left(p, x_{2}\right) \leq d\left(p, f\left(x_{1}\right)\right)+d\left(f\left(x_{1}\right), x_{2}\right)<$ $\lambda d\left(p, x_{1}\right)+\delta<\delta(\lambda+1)$. Thus we obtain

$$
\begin{aligned}
d\left(p, x_{i}\right) & \leq d\left(p, f\left(x_{i-1}\right)\right)+d\left(f\left(x_{i-1}\right), x_{i}\right)<d\left(p, f\left(x_{i-1}\right)\right)+\delta \\
& <\lambda d\left(p, x_{i-1}\right)+\delta<\cdots<\delta\left(1+\lambda+\cdots+\lambda^{i}\right) \\
& \leq \frac{\delta}{1-\lambda} .
\end{aligned}
$$

Put $\eta=\delta /(1-\lambda)$. Then if $\delta$ is sufficiently small then we can make $\eta<\epsilon$. This is a contradiction since $d(y, p) \geq 2 \epsilon$. 
Theorem 2.2 (Corollary 2.19 in [12]) For any $\epsilon>0$, there are two integers $l$ and $n$ such that for any periodic point $x$ of period $p(x) \geq n$ :

(1) either $f$ admits an l-dominated splitting along the orbit of $x$;

(2) or, for any neighborhood $U$ of the orbit of $x$, there exists an $\epsilon$-perturbation $g$ off in the $C^{1}$-topology, coinciding with $f$ outside $U$ and on the orbit of $x$, and such that $x$ is a source or a sink of $g$ for which the differential $D_{x} g^{p(x)}$ has all eigenvalues real with the same modulus.

We say that the Hausdorff distance between two closed subsets $A$ and $B$ of $M$ is given by

$$
d_{H}(A, B)=\max \left\{\sup _{x \in A} d(x, B), \sup _{y \in B} d(A, y)\right\} .
$$

Lemma 2.3 (Theorem 4 in [9]) There is a residual set $\mathcal{G} \subset \operatorname{Diff}(M)$ such that for any $f \in \mathcal{G}$, a compact invariant set $\Lambda$ is the Hausdorfflimit of a sequence of periodic points if and only if $\Lambda$ is chain transitive.

Lemma 2.4 If $\left.\right|_{\Lambda}$ is robustly chain transitive, then $\Lambda$ admits a dominated splitting.

Proof Let $\left.f\right|_{\Lambda}$ be robustly chain transitive. Then there are a $C^{1}$-neighborhood $\mathcal{U}(f)$ of $f$ and a neighborhood $U$ of $\Lambda$ such that for any $g \in \mathcal{U}(f),\left.g\right|_{\Lambda_{g}(U)}$ is chain transitive. By Lemma 2.3, take $\mathcal{U}_{0}(f)=\mathcal{U}(f) \cap \mathcal{G}$. Then there exist $f_{n} \in \mathcal{U}_{0}(f)$ with $f_{n} \rightarrow f$ and $\operatorname{Orb}_{f_{n}}\left(p_{n}\right)$ of $f_{n}$ such that

$$
\Lambda=\limsup _{n \rightarrow \infty} \operatorname{Orb}_{f_{n}}\left(p_{n}\right)
$$

Since $\left.f\right|_{\Lambda}$ is robustly chain transitive, by Lemma $2.1,\left.f\right|_{\Lambda}$ does not contains neither sinks nor sources. By Theorem 2.2, $f_{n}$ admits an $l$-dominated splitting over $\operatorname{Orb}_{f_{n}}\left(p_{n}\right)$ with $l$ independent $n$. Thus $\Lambda$ admits an $l$-dominated splitting.

By Mañé (see [6]), the family of periodic sequences of linear isomorphisms of $\mathbb{R}^{\mathrm{dim} M}$ generated by $D g$ ( $g$ close to $f$ ) along the hyperbolic periodic point $q \in \Lambda_{g}(U) \cap P(g)$ is uniformly hyperbolic. This means that there is $\epsilon>0$ such that for any $g C^{1}$-nearby $f, q \in$ $\Lambda_{g}(U) \cap P(g)$ and any sequence of linear maps $A_{i}: T_{g^{i}(q)} M \rightarrow T_{g^{i+1}(q)} M$ with $\left\|A_{i}-D_{g^{i}(q)} g\right\|<$ $\epsilon(i=1,2, \ldots, \pi(q)), \prod_{i=0}^{\pi(q)-1} A_{i}$ is hyperbolic. By Proposition II.1 in [6], we have the following.

Lemma 2.5 Suppose that there is a $C^{1}$-neighborhood $\mathcal{U}(f)$ off such that for any $g \in \mathcal{U}(f)$, any periodic point of $\Lambda_{g}(U)$ is hyperbolic. Then there are constants $C>0,0<\lambda<1$ and $m>0$ such that iffor any $p \in \Lambda_{g}(U) \cap P(g)$ has minimum period $\pi(p) \geq m$ then

$$
\begin{aligned}
& \prod_{i=0}^{k-1}\left\|\left.D_{g^{i m}(p)} g^{m}\right|_{E^{s}\left(g^{i m}(p)\right)}\right\|<C \lambda^{k} \quad \text { and } \\
& \prod_{i=0}^{k-1}\left\|\left.D_{g^{-i m}(p)} g^{m}\right|_{E^{u}\left(g^{-i m}(p)\right)}\right\|<C \lambda^{k},
\end{aligned}
$$

where $k=[\pi(p) / m]$. 
Let us recall Mañés ergodic closing lemma in [6]. For any $\epsilon>0$, let $B_{\epsilon}(f, x)$ an $\epsilon$-tubular neighborhood of $f$-orbit of $x$, i.e., $B_{\epsilon}(f, x)=\left\{y \in M: d\left(f^{n}(x), y\right)<\epsilon\right.$ for some $\left.n \in \mathbb{Z}\right\}$. Let $\Sigma_{f}$ be the set of points $x \in M$ such that for any $C^{1}$-neighborhood $\mathcal{U}(f)$ of $f$ and $\epsilon>0$, there are $g \in \mathcal{U}(f)$ and $y \in P(g)$ satisfying $g=f$ on $M \backslash B_{\epsilon}(f, x)$ and $d\left(f^{i}(x), g^{i}(y)\right) \leq \epsilon$ for $0 \leq i \leq \pi(y)$.

Remark 2.6 (Theorem A in [6]) For any $f$-invariant probability measure $\mu$, we have $\mu\left(\Sigma_{f}\right)=1$.

Proof of Theorem 1.1 By assumption, $\left.f\right|_{\Lambda}$ is robustly chain transitive. Then by Lemma 2.4, $\Lambda$ admits a dominated splitting $E \oplus F$. We will finish the proof of Theorem 1.1, we show that

$$
\liminf _{n \rightarrow \infty}\left\|\left.D_{x} f^{n}\right|_{E_{x}}\right\|=0 \quad \text { and } \quad \liminf _{n \rightarrow \infty}\left\|D_{x} f^{-n}{\mid F_{x}}_{x}\right\|=0
$$

for all $x \in \Lambda$. Then the splitting is hyperbolic. To prove, we consider $\liminf _{n \rightarrow \infty}\left\|D_{x} f_{\mid E}^{n}\right\|=0$ (other case is similar). It is enough to show that for any $x \in \Lambda$, there exists $n=n(x)>0$ such that

$$
\prod_{j=0}^{n-1}\left\|\left.D f^{m}\right|_{E_{f} m j(x)}\right\|<1 .
$$

We will derive a contraction. If it is not true, then there is $x \in \Lambda$ such that

$$
\prod_{j=0}^{n-1}\left\|\left.D f^{m}\right|_{E_{f^{m j}(x)}}\right\| \geq 1
$$

for all $n \geq 0$. Thus

$$
\frac{1}{n} \sum_{j=0}^{n-1} \log \left\|\left.D f^{m}\right|_{E_{f^{m j}(x)}}\right\| \geq 0
$$

for all $n \geq 0$. Define a probability measure

$$
\mu_{n}:=\frac{1}{n} \sum_{j=0}^{n-1} \delta_{f^{j}(x)}
$$

Then there exists $\mu_{n_{k}}(k \geq 0)$ such that $\mu_{n_{k}} \rightarrow \mu_{0} \in \mathcal{M}_{f}(M)$, as $k \rightarrow \infty$, where $M$ is compact metric space. Thus

$$
\begin{aligned}
\int \log \left\|\left.D f\right|_{E_{x}}\right\| d \mu_{0} & =\lim _{k \rightarrow \infty} \int \log \left\|\left.D f\right|_{E_{x}}\right\| d \mu_{n_{k}} \\
& =\lim _{k \rightarrow \infty} \frac{1}{n} \sum_{j=0}^{n-1} \log \left\|\left.D f\right|_{E_{f^{\prime}(x)}}\right\| \geq 0 .
\end{aligned}
$$

By Mañé ([6], p.521),

$$
\int_{\Lambda} \log \left\|\left.D f\right|_{E_{x}}\right\| d \mu_{0}=\int_{\Lambda} \frac{1}{n} \sum_{j=0}^{n-1} \log \left\|\left.D_{f^{\prime}(x)} f\right|_{E_{f^{\prime}(x)}}\right\| d \mu_{0} \geq 0
$$


where $\mu_{0}$ is a $f$-invariant measure. Let

$$
B_{\epsilon}(f, x)=\left\{y \in M: d\left(f^{n}(x), y\right)<\epsilon \text { for some } n \in \mathbb{Z}\right\},
$$

and $\Sigma_{f}=\left\{x \in M: d\left(f^{n}(x), y\right)<\epsilon\right.$, there exist $g \in \mathcal{U}(f)$ and $y \in P(g)$ such that $g=f$ on $M \backslash$ $B_{\epsilon}(f, x)$ and $d\left(f^{i}(x), f^{i}(y)\right) \leq \epsilon$ for $\left.0 \leq i \leq \pi(y)\right\}$.

For any $\mu \in \mathcal{M}_{f}(M), \mu\left(\Sigma_{f}\right)=1$. Then, for any $\mu \in \mathcal{M}_{f}(\Lambda)$,

$$
\mu\left(\Lambda \cap \Sigma_{f}\right)=1,
$$

since $\mu(\Lambda)=1$ and $\mu\left(\Sigma_{f}\right)=1$. Thus, $\Lambda=\Lambda \cap \Sigma(f)$ almost everywhere. Therefore,

$$
\int_{\Lambda \cap \Sigma(f)} \lim _{n \rightarrow \infty} \frac{1}{n} \sum_{j=0}^{n-1} \log \left\|\left.D f\right|_{E_{f^{\prime}(x)}}\right\| d \mu \geq 0 .
$$

By Birkhoff's theorem, and the ergodic closing lemma, we can take $z_{0} \in \Lambda \cap \Sigma(f)$ such that

$$
\lim _{n \rightarrow \infty} \frac{1}{n} \sum_{j=0}^{n-1} \log \left\|\left.D f\right|_{E_{f} j_{\left(z_{0}\right)}}\right\| \geq 0 .
$$

By Lemma 2.5 , this is a contradiction. Thus by Lemma $2.5, z_{0} \notin P(f)$.

Let $C>0, m>0$, and $\lambda \in(0,1)$ be given by Lemma 2.5 and take $\lambda<\lambda_{0}<1$ and $n_{0}>0$ such that

$$
\frac{1}{n} \sum_{j=0}^{n-1} \log \left\|\left.D f^{m}\right|_{E_{f^{m} j_{\left(z_{0}\right)}}}\right\| \geq \log \lambda_{0}, \quad \text { if } n \geq n_{0} .
$$

Then, by Mañés ergodic closing lemma, we can find $g \in \mathcal{V}_{0}(f), g=f$ on $M \backslash U_{j}$ and $r_{g} \in$ $\Lambda_{g} \cap P(g)$ near by $z_{0}$. By assumption, for any $p \in \Lambda_{g}(U) \cap P(g)$ we know that

$$
\operatorname{index}\left(r_{g}\right)=\operatorname{index}(p)
$$

since $g=f$ on $M \backslash U_{j}$. Since $\left.f\right|_{\Lambda}$ is robustly chain transitive, we can construct $h \in \mathcal{V}_{0}(f)$ $(\subset \mathcal{V}(f)) C^{1}$-nearby $g$ such that

$$
\lambda_{0}^{k} \leq \prod_{i=0}^{k-1}\left\|\left.D_{h^{i m}\left(r_{h}\right)} h^{m}\right|_{E_{h^{i m}\left(r_{h}\right)}}\right\|
$$

(see [6], pp.523-524). On the other hand, by Lemma 2.5, we see that

$$
\prod_{i=0}^{k-1}\left\|\left.D_{h^{i m}\left(r_{h}\right)} h^{m}\right|_{E_{h^{i m}\left(r_{h}\right)}}\right\|<C \lambda^{k} .
$$

We can choose the period $\pi\left(r_{h}\right)\left(>n_{0}\right)$ of $r_{h}$ as large as $\lambda_{0}^{k} \geq C \lambda^{k}$. Here $k=\left[\pi\left(r_{h}\right) / m\right]$. This is a contradiction. Thus,

$$
\liminf _{n \rightarrow \infty}\left\|\left.D_{x} f^{n}\right|_{E_{x}}\right\|=0
$$

for all $x \in \Lambda$. Therefore, $\Lambda$ is hyperbolic. This completes the proof of Theorem 1.1. 
Competing interests

The author declares to have no competing interests.

\section{Acknowledgements}

ML is supported by Basic Science Research Program through the National Research Foundation of Korea (NRF) funded by the Ministry of Science, ICT \& Future Planning (No. 2014R1A1A1A05002124).

Received: 29 December 2014 Accepted: 9 July 2015 Published online: 23 July 2015

\section{References}

1. Hayashi, S: Diffeomorphisms in $\mathcal{F}^{1}(M)$ satisfy Axiom A. Ergod. Theory Dyn. Syst. 12, 233-253 (1992)

2. Mañé, R: A proof of the $C^{1}$ stability conjecture. Publ. Math. Inst. Hautes Études Sci. 66, 161-210 (1988)

3. Robbin, J: A structural stability theorem. Ann. Math. 94, 447-493 (1971)

4. Robinson, C: Structural stability of $C^{1}$ diffeomorphisms. J. Differ. Equ. 22, 28-73 (1976)

5. Mañé, R: Contributions to stability conjecture. Topology 17, 383-396 (1978)

6. Mañé, R: An ergodic closing lemma. Ann. Math. 116, 503-540 (1982)

7. Díaz, LJ, Pujals, E, Ures, R: Partial hyperbolicity and robust transitivity. Acta Math. 183, 1-43 (1999)

8. Bonatti, C, Díaz, LJ, Pujals, E: A C $C^{1}$-generic dichotomy for diffeomorphisms: weak forms of hyperbolicity or infinitely many sinks or sources. Ann. Math. 158, 187-222 (2003)

9. Crovisier, S: Periodic orbits and chain-transitive sets of $C^{1}$ diffeomorphisms. Publ. Math. Inst. Hautes Études Sci. 104, 87-141 (2006)

10. Robinson, C: Dynamical Systems: Stability, Symbolic Dynamics, and Chaos, 2nd edn. Studies in Advanced Mathematics. CRC Press, Boca Raton (1999)

11. Arbieto, A, Ribeiro, R: Flows with the (asymptotic) average shadowing property on three-dimensional closed manifolds. Dyn. Syst. 26, 425-432 (2011)

12. Bonatti, C, Gourmelon, N, Vivier, T: Perturbations of the derivative along periodic orbits. Ergod. Theory Dyn. Syst. 26, 1307-1337 (2006)

\section{Submit your manuscript to a SpringerOpen ${ }^{\circ}$ journal and benefit from:}

- Convenient online submission

Rigorous peer review

- Immediate publication on acceptance

- Open access: articles freely available online

- High visibility within the field

- Retaining the copyright to your article 